Computer Physics Communications 15 (1978) 259-273

(c) North-Holland Publishing Company

\title{
II. REDUCED SU(3) MATRIX ELEMENTS *
}

\section{BRAUNSCHWEIG **}

Dept. of Physics, University of Michigan, Ann Arbor, Michigan 48109, USA

Received 8 February 1978

\section{PROGRAM SUMMARY}

Title of program: REDUCED SU(3) MATRIX ELEMENTS

Catalogue number: ABKH

Program obtainable from: CPC Program Library, Queen's University of Belfast, N. Ireland (see application form in this issue)

Computer: AMDAHL 470V/6, IBM 360/370; Installation: Univ. of Michigan, Ann Arbor, Mich.

Operating system: MTS

Program language used: FORTRAN

High speed storage required: 212 kwords

No. of bits in a word: 32

Overlay structure: none

No. of magnetic tapes required: none

Other peripherals used: card reader, line printer (card punch)

No. of cards of combined program and test deck: 907

Card punching code: EBCDIC

CPC library programs used:

Catalogue number: $\mathrm{ABKG}$

Title: REDUCED SU(3) CFPS

Ref. in CPC: 14 (1978) 109

Catalogue number: AAC*

Title: DATA FOR ABKG

Ref. in CPC: 14 (1978) 109

* Work supported by the U.S. National Science Foundation.

** Present address: Goddard Space Flight Center, Seabrook, MD, USA. 
Keywords: Nuclear physics, SU(3), SU(4), shell model, matrix elements, nuclear structure, pseudo-SU(3), one-body operators, two-body operators, theoretical methods

\section{Nature of physical problem}

Reduced SU(3) matrix elements (RME) are calculated for a general one or two body tensor operator, between arbitrary shell model states in an SU(3) $\times \mathrm{SU}(4)$ or SU(3) $\times \mathrm{SU}(2)$ scheme. Matrix elements in the angular momentum scheme can then be obtained by multiplying these RME with the appropriate SU(3) $\supset \mathrm{R}(3)$ coupling coefficients available through the code of ref. [2].

\section{Method of solution}

One and two body tensor operators are explicitly constructed in terms of fermion creation operators. Since the $\langle\mathrm{BRA}|$ and $|K E T\rangle$ states obtained by using the appropriate routines of the code of ref. [1] are also written in terms of fermion creation operators, the overlap can be calculated directly. This method is typically $2-3$ orders or magnitude faster than the usual CFP expansion.

\section{Restrictions on the complexity of the problem}

The $\langle\mathrm{BRA}|$ and $|\mathrm{KET}\rangle$ states are restricted to those that can be calculated with the code of ref. [1]. No other restrictions exist.

\section{Typical running time}

It is a critical function of the nuclear shell, the number of particles as well as the options selected.

\section{Unusual features of the program}

The same array naming convention adopted in the code of ref. [1] is kept. This allows a saving of up to $35 \%$ of high speed storage in IBM $360 / 370$ or similar operating systems.

References

[1] D. Braunschweig, Comput. Phys. Commun. 14 (1978) 109.

[2] Y. Akiyama and J.P. Draayer, Comput. Phys. Commun. 5 (1973) 405.

\section{LONG WRITE-UP}

\section{Introduction}

Conventional computer codes [3] for shell model calculations are limited to the $j-j$ coupling scheme, and even though these can handle matrices of very large size the types of problems which can be tackled are very limited. To date detailed calculations [4] have been carried out only for many nucleon systems in the $p_{1 / 2} p_{3 / 2}$ and $s_{1 / 2} d_{3 / 2} d_{5 / 2}$ (or $p_{1 / 2} p_{3 / 2} f_{5 / 2}$ ) shells with no excitations out of the "valence" shell.

Often an underlying symmetry can be used to reduce the size of the shell model matrix. For some nuclei SU(3) symmetry [5] (or pseudo SU(3) symmetry) [6] may make a severe truncation of the shell model matrix physically meaningful. In a nucleus of good SU(3) symmetry the low-lying nuclear states may be approximated very well in terms of a small number (typically 3-6) of irreducible representations of SU(3). In such a nucleus therefore a shell model calculation in the SU(3) scheme may be characterized by a small basis.

The main difficulty in performing SU(3) shell model calculations is then concentrated in the calculation of matrix elements of one and two body operators in this basis.

We describe below a FORTRAN program which calculates reduced matrix elements (RME) directly, that is, without using the usual coefficient of fractional parentage (CFP) expansion (see eqs. (16) and (17) of ref. [7]). This results in a speed increase of $2-3$ orders of magnitude.

The full matrix element can then be obtained by multiplying the RME by the appropriate $S U(3) \supset R(3)$ and $R(3) \supset R(2)$ Wigner coefficients available through the code of ref. [2].

Although the present program is restricted to the calculation of RME for many-particle shell model states with particles of the same shell only; the combination of the codes for RME's of one-body operators with the CFP code of ref. [1] and straightforward recoupling techniques' make it possible to calculate matrix elements which involve 
particle-hole excitations. However, the CFP expansion needed for this purpose is simple, since a two-body operator can involve at most 2-particle excitations even if the basis includes complicated core excitations.

The RME program calls several routines from the codes ABKG [1] and ACRM [2] henceforth called CFP PROGRAM and SU(3) PACKAGE respectively. Familiarity with the CFP PROGRAM is assumed. Care has been exercised to maintain the naming and dimensioning conventions adopted for the CFP PROGRAM. Generality is preserved as well, in that the RME program allows calculations to be performed in:

1) Any major oscillator shell.

2) Both neutron-proton formalism (NPF) or full spin-isospin formalism (FSIF) are acceptable.

3) Optimization available for the prolate or oblate limit is obtained by calculating the RME from the highest weight state or lowest weight state, respectively.

4) No SU(4) coupling coefficients are needed since both $\langle\mathrm{BRA}|$ and $|\mathrm{KET}\rangle$ states can be lowered in SU(4) according to the needs of a particular user.

5) Arbitrary one and two body tensor operators of good SU(3) symmetry in either the NPF or FSIF are acceptable.

\section{Method of calculation}

Throughout this section we will use the notation introduced in ref. [1]; furthermore we will illustrate our method using the full spin-isospin formalism.

The calculation of the RME proceeds in the following order:

1) Explicit construction of the many-particle states in terms of fermion creation operators.

2) Explicit construction of the desired tensor operators in terms of fermion creation and destruction operators.

3) The tensor operators constructed in 2) are allowed the act on the $|\mathrm{KET}\rangle$ states and the result is overlapped with the $\langle$ BRA | states.

4) Finally the $S U(3) \supset S U(2) \times U(1)$ and $S U(2) \supset U(1)$ dependence is factored out from the overlap by using the appropriate Wigner coefficients.

\subsection{Construction of the may-particle states}

The many-particle states are classified according to the IR of SU(3) $\times$ SU(4). The subgroup chains used are $\mathrm{SU}(3) \supset \mathrm{SU}(2) \times \mathrm{U}(1)$ and $\mathrm{SU}(4) \supset[\mathrm{SU}(2) \times \mathrm{SU}(2)]$. Since the many-particle states are highest weight in SU(3) and $[\mathrm{SU}(2) \times \mathrm{SU}(2)]$ they can be denoted by:

$$
\left|n(\lambda \mu) \alpha\left(\mathrm{PP}^{\prime} P^{\prime \prime}\right) \beta S T\right\rangle
$$

where $n=$ number of particles,

$(\lambda \mu) \quad=\mathrm{SU}(3)$ irreducible representation,

$\alpha \quad=$ inner multiplicity label for SU(3) needed to distinguish multiple occurrence of $(\lambda \mu)$ in a given space symmetry; $\alpha=1,2, \ldots, \alpha_{\max }$,

$\left(\mathrm{PP}^{\prime} \mathrm{P}^{\prime \prime}\right)=$ supermultiplet labels,

$S \quad=$ spin,

$T \quad=$ isospin,

$\beta \quad=$ inner multiplicity label for SU(4) needed to distinguish multiple occurrences of $S T$ in a given supermultiplet; $\beta=1,2, \ldots, \beta_{\max }$.

The method used to construct these many-particle states is spelled out in detail in sections 2.1 through 2.4 of Ití. [1]. The highest weight states of (1) can be expressed in terms of fermion creation operators as:

$$
\left|n(\lambda \mu) \alpha\left(\mathrm{PP}^{\prime} \mathrm{P}^{\prime \prime}\right) \beta S T\right\rangle=\sum_{i_{1}<i_{2}<\ldots<i_{m}} Z_{i_{1} i_{2} \ldots i_{m}}^{(\lambda \mu) \alpha\left(\mathrm{PP}^{\prime} \mathrm{P}^{\prime \prime}\right) \beta S T} a_{i_{1}}^{+} a_{i_{2}}^{+} \ldots a_{i_{m}}^{+}|0\rangle
$$


where $i_{j}$ is an index which labels the single-particle levels (see fig. 1 of ref. [1]).

The single-particle fermion creation operator have tensorial character given by:

$$
\begin{array}{r}
a_{\left(\eta_{0}\right) \epsilon \Lambda M_{\Lambda} \frac{1}{2} M_{s} \frac{1}{2} M_{T}}^{+}=T^{\left(\eta_{0}\right) \epsilon \Lambda \frac{1}{2} \frac{1}{2}} \\
M_{\Lambda} M_{S} M_{T}
\end{array},
$$

where $\eta$ is the number of oscillator quanta in the shell under consideration; similarly the tensorial character of the single-particle destruction operators is given by:

$$
a_{\left(\eta_{0}\right) \in \Lambda M_{\Lambda} \frac{1}{2} M_{S} \frac{1}{2} M_{T}}=(-)^{\eta+\Lambda-M_{\Lambda}+\frac{1}{2}-M_{S}+\frac{1}{2}-M_{T}} T_{\left.-M_{\Lambda}-M_{S}-M_{T}\right) \in \Lambda}^{\frac{1}{2}} \frac{1}{2} .
$$

\subsection{Construction of one and two body tensor operators}

In this section we illustrate the construction of two body tensor operators. One body operators are obtained in a similar fashion.

We denote a two particle creation operator by:

$$
\left[a^{+} a^{+}\right]^{(\lambda \mu) \in \Lambda M_{\Lambda} S M_{S} T M_{T}}
$$

where

$(\lambda \mu)=\operatorname{SU}(3) \mathrm{IR}$,

$\epsilon \Lambda M_{\Lambda}=$ subgroup labels of $\mathrm{SU}(3)$ belonging to $\mathrm{SU}(2) \times \mathrm{U}(1)$,

$S \quad=$ spin,

$M_{S} \quad=Z$-projection of $S$,

$T \quad=$ isospin,

$M_{T} \quad=Z$-projection of $T$.

The square brackets $[\ldots]$ denote $\mathrm{SU}(3)$ as well as spin and isospin coupling. Then the two body operator of arbitrary tensorial chracter can be expressed as:

$$
\begin{aligned}
& {\left[\left[a^{+} a^{+}\right]^{\left(\lambda_{1} \mu_{1}\right) S_{1} T_{1}}[a a]^{\left(\mu_{2} \lambda_{2}\right) S_{2} T_{2}}\right]^{(\lambda \mu) \rho_{12} \epsilon \Lambda M_{\Lambda} S M_{S} T M_{T}}=\sum_{\substack{\epsilon_{1} \Lambda_{1} \Lambda_{2} \\
M_{\Lambda_{1}} M_{S_{1} M_{T_{1}}}}}(-)^{\frac{1}{3}\left(\mu_{2}-\lambda_{2}\right)+\frac{1}{6} \epsilon_{2}-M_{\Lambda_{2}}+S_{2}-M_{S_{2}}+T_{2}-M_{T_{2}}}} \\
& \left\langle\left(\lambda_{1} \mu_{1}\right) \epsilon_{1} \Lambda_{1} ;\left(\mu_{2} \lambda_{2}\right) \epsilon_{2} \Lambda_{2} \|(\lambda \mu) \epsilon \Lambda\right\rangle_{\rho_{12}}\left\langle\Lambda_{1} M_{\Lambda_{1}} \Lambda_{2} M_{\Lambda_{2}} \mid \Lambda M_{\Lambda}\right\rangle\left\langle S_{1} M_{S_{1}} S_{2} M_{S_{2}} \mid S M_{S}\right\rangle\left\langle T_{1} M_{T_{1}} T_{2} M_{T_{2}} \mid T M_{T}\right\rangle,
\end{aligned}
$$

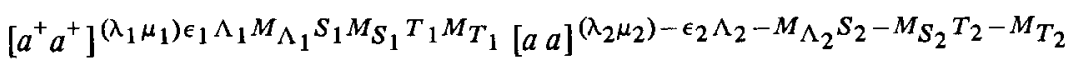

$$
\begin{aligned}
& =\sum_{i_{1}<i_{2}, i_{3}<i_{4}} X_{i_{1} i_{2} i_{3} i_{4}}^{\left[\left(\lambda_{1} \mu_{1}\right) S_{1} T_{1}\left(\mu_{2} \lambda_{2}\right) S_{2} T_{2}\right]}{ }^{(\lambda \mu)_{\rho_{12}} \in \Lambda M_{\Lambda} S M_{S} T M_{T}} a_{i_{1}}^{+} a_{i_{2}}^{+} a_{i_{3}} a_{i_{4}},
\end{aligned}
$$

where the double barred coefficient is a reduced $\mathrm{SU}(3) \supset \mathrm{SU}(2) \times \mathrm{U}(1)$ Wigner coefficient and the single barred coefficients are ordinary Clebsh-Gordan coefficients.

$\rho_{12}$ labels multiple occurrence of $(\lambda \mu)$ in the Kronecker product $\left(\lambda_{1} \mu_{1}\right) \times\left(\mu_{2} \lambda_{2}\right)$; this label is not needed for one body operators.

The two particle operators of (2) need not be calculated since they are read in as input data from set A (see section 4.1.1).

\subsection{Calculation of the overlap}

If we let the operator of eq. (5) act on the $|\mathrm{KET}\rangle$ of eq. (1) we get:

$$
\begin{aligned}
& {\left[\left[a^{+} a^{+}\right]^{\left(\lambda_{1} \mu_{1}\right) S_{1} T_{1}}[a a]^{\left(\mu_{2} \lambda_{2}\right) S_{2} T_{2}}\right]^{(\lambda \mu)} \rho_{12} \in \Lambda M_{\Lambda} S M_{S} T M_{T}\left|n\left(\lambda_{\mathrm{k}} \mu_{\mathrm{k}}\right) \alpha_{\mathrm{k}}\left(\mathrm{P}_{\mathrm{k}} \mathrm{P}_{\mathrm{k}}^{\prime} \mathrm{P}_{\mathrm{k}}^{\prime \prime}\right) \beta_{\mathrm{k}} S_{\mathrm{k}} T_{\mathrm{k}}\right\rangle}
\end{aligned}
$$

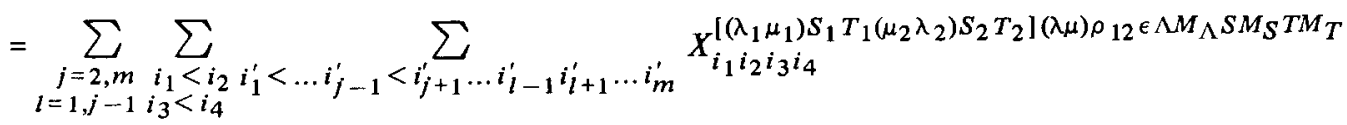




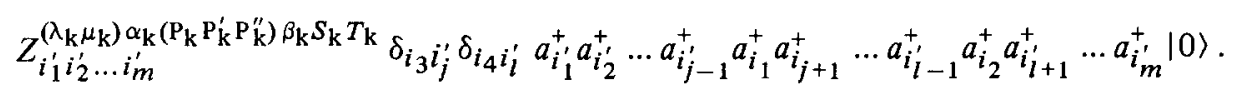

If $i_{1}<i_{j-1}^{\prime}$ or $i_{1}>i_{j+1}^{\prime}$ or $i_{2}<i_{l-1}^{\prime}$ or $i_{2}>i_{l+1}^{\prime}$ we have to reorder the fermion operators, this introduces a phase factor $P$. The final result can then be written as:

$$
\sum_{i_{1}^{\prime \prime}<i_{2}^{\prime \prime}<\ldots i_{m}^{\prime \prime}} X^{0} Z^{\mathrm{k}} P^{\mathrm{k}} a_{i_{1}^{\prime \prime}}^{+} a_{i_{2}^{\prime \prime}}^{+} \ldots a_{i_{m}^{\prime \prime}}^{+}
$$

where we have replaced the quantum numbers associated with $X$ by the superscript " 0 " and the quantum numbers associated with $Z$ by a superscript "k".

We can now evaluate the overlap $0_{\Lambda}$ as:

$$
\begin{aligned}
0_{\Lambda} & =\left\langle n\left(\lambda_{\mathrm{b}} \mu_{\mathrm{b}}\right) \alpha_{\mathrm{b}}\left(\mathrm{P}_{\mathrm{b}} \mathrm{P}_{\mathrm{b}}^{\prime} \mathrm{P}_{\mathrm{b}}^{\prime \prime}\right) \beta_{\mathrm{b}} S_{\mathrm{b}} T_{\mathrm{b}}\left[\left[a^{+} a^{+}\right]^{\left(\lambda_{1} \mu_{1}\right) S_{1} T_{1}}[a a]^{\left(\mu_{2} \lambda_{2}\right) S_{2} T_{2}}\right]^{(\lambda \mu) \rho_{12} \in \Lambda M_{\Lambda} S M_{S} T M_{T}}\right. \\
& \left.\ln \left(\lambda_{\mathrm{k}} \mu_{k}\right) \alpha_{\mathrm{k}}\left(\mathrm{P}_{\mathrm{k}} \mathrm{P}_{\mathrm{k}}^{\prime} \mathrm{P}_{\mathrm{k}}^{\prime \prime}\right) \beta_{\mathrm{k}} S_{\mathrm{k}} T_{\mathrm{k}}\right\rangle \\
& =\sum_{i^{\prime \prime} i^{\prime \prime}} Z^{\mathrm{b}} X^{0} Z^{\mathrm{k}} P^{\mathrm{k}}\left\langle 0\left|a_{i_{m}^{\prime \prime \prime}} \ldots a_{i_{1}^{\prime \prime \prime}} a_{i_{1}^{\prime \prime}}^{+} \ldots a_{i_{m}^{\prime \prime}}^{+}\right| 0\right\rangle=\sum_{i^{\prime \prime} i^{\prime \prime}} Z^{\mathrm{b}} Z^{0} Z^{\mathrm{k}} P^{\mathrm{k}} \delta_{i^{\prime \prime} i^{\prime \prime}}
\end{aligned}
$$

Since the $\langle$ BRA $|$ and $|K E T\rangle$ states are highest weight in SU(3), the total number of admissible values of $\Lambda$ is equal to $\rho_{\max }$, the number of times $\left(\lambda_{\mathrm{b}} \mu_{\mathrm{b}}\right)$ is contained in the Kronecker product $\left(\lambda_{\mathrm{k}} \mu_{\mathrm{k}}\right) \times(\lambda \hat{\mu})$.

The RME is then obtained by solving the system of linear equations:

$$
\begin{aligned}
& 0_{\Lambda}=\sum_{\rho=1}^{\rho_{\max }}\left\langle\left(\lambda_{\mathrm{k}} \mu_{\mathrm{k}}\right) \epsilon_{\mathrm{k}} \Lambda_{\mathrm{k}} ;(\lambda \mu) \epsilon \Lambda \|\left(\lambda_{\mathrm{b}} \mu_{\mathrm{b}}\right) \epsilon_{\mathrm{b}} \Lambda_{\mathrm{b}}\right\rangle_{\rho}\left\langle\Lambda_{\mathrm{k}} \Lambda_{\mathrm{k}} \Lambda M_{\Lambda} \mid \Lambda_{\mathrm{b}} \Lambda_{\mathrm{b}}\right\rangle\left\langle S_{\mathrm{k}} S_{\mathrm{k}} S M_{S} \mid S_{\mathrm{b}} S_{\mathrm{b}}\right\rangle\left\langle T_{\mathrm{k}} T_{\mathrm{k}} T M_{T} \mid T_{\mathrm{b}} T_{\mathrm{b}}\right\rangle \\
& \left.\left\langle n\left(\lambda_{\mathrm{b}} \mu_{\mathrm{b}}\right) \alpha_{\mathrm{b}}\left(\mathrm{P}_{\mathrm{b}} \mathrm{P}_{\mathrm{b}}^{\prime} \mathrm{P}_{\mathrm{b}}^{\prime \prime}\right) \beta_{\mathrm{b}} S_{\mathrm{b}} T_{\mathrm{b}} \|||\left[a^{+} a^{+}\right]^{\left(\lambda_{1} \mu_{1}\right) S_{1} T_{1}}[a a]^{\left(\lambda_{2} \mu_{2}\right) S_{2} T_{2}}\right]^{(\lambda \mu) \rho_{12} S T} \| n\left(\lambda_{\mathrm{k}} \mu_{\mathrm{k}}\right) \alpha_{\mathrm{k}}\left(\mathrm{P}_{\mathrm{k}} \mathrm{P}_{\mathrm{k}}^{\prime} \mathrm{P}_{\mathrm{k}}^{\prime \prime}\right) \beta_{\mathrm{k}} S_{\mathrm{k}} T_{\mathrm{k}}\right\rangle_{\rho},
\end{aligned}
$$

where

$$
\epsilon_{\mathrm{k}}=2 \lambda_{\mathrm{k}}+\mu_{\mathrm{k}}, \quad \Lambda_{\mathrm{k}}=\mu_{\mathrm{k}} / 2, \quad \epsilon_{\mathrm{b}}=2 \lambda_{\mathrm{b}}+\mu_{\mathrm{b}}, \quad \Lambda_{\mathrm{b}}=\mu_{\mathrm{b}} / 2 .
$$

The triple barred quantity in eq. (9) is the RME.

\section{Structure of the program}

The overall structure of the RME program is illustrated in the block diagram of fig. 1.

Block 1 contains a MAIN routine as well as subroutine RME and subroutine TENSOR. These are the only routines provided as part of the RME program; their function is described in detail in comment statements at the beginning of each routine.

In block 2 the explicit construction of the states of eq. (2) is performed. All the subroutines in block 2 are part of the CFP program; we therefore do not provide them as part of the RME code; they can be obtained from the CPC library (code number ABKG). The subroutines which are enclosed in dotted square in fig. 1 need only be included for calculations in the FSIF.

In block 3 the $\mathrm{SU}(3) \supset \mathrm{SU}(2) \times \mathrm{U}(1)$ and the $\mathrm{SU}(2) \supset \mathrm{U}(1)$ Wigner coefficients are calculated. These are needed for eq. (5) and eq. (9). All the subroutines in block 3 are part of the SU(3) PACKAGE but for convenience they were repeated as part of the deck ABKG and can be obtained from it.

Communication between different blocks is established through labelled COMMON blocks. The meaning of the variables in a given block is explained in comment cards at the beginning of those subroutines which need this common block for communication. 


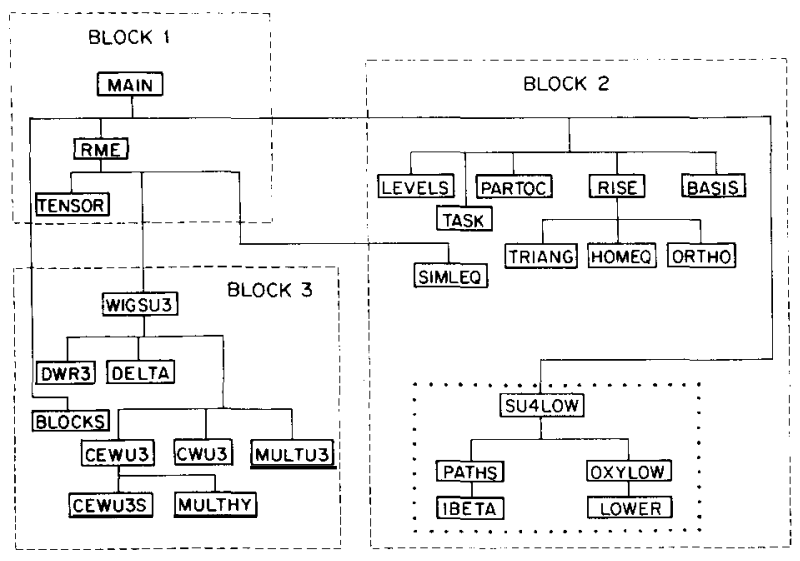

Fig. 1. Flow diagram for program reduced SU(3) matrix elements.

Because of implicit dimensioning in the subroutines of block 3 it is important to have these routines follow those of block 1 and 2 in the source deck.

\section{Input/output}

Input to the RME program is done exclusively in the MAIN routine. The structure of the input data deck is illustrated in fig. 2. The input data is of two types as we describe below.

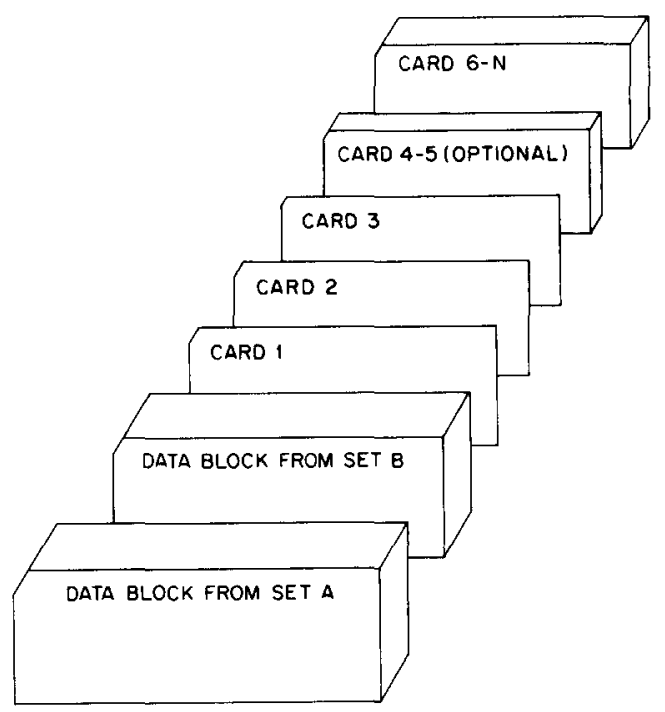

Fig. 2. Structure of the input data deck. 
Table 1

FSIF

\begin{tabular}{llcc}
\hline Operator & Shell & First card & Last card \\
\hline 1-body & $\mathrm{p}$ & 1 & 13 \\
2-body & $\mathrm{p}$ & 14 & 74 \\
1-body & $\mathrm{sd}$ & 151 & 172 \\
2-body & $\mathrm{sd}$ & 173 & 451 \\
1-body & $\mathrm{fp}$ & 1876 & 1908 \\
2-body & $\mathrm{fp}$ & 1909 & 2881 \\
\hline NPF & & & \\
\hline 1-body & $\mathrm{p}$ & 6045 & 6056 \\
2-body & $\mathrm{p}$ & 6057 & 6081 \\
1-body & $\mathrm{sd}$ & 6082 & 6094 \\
2-body & $\mathrm{sd}$ & 6095 & 6164 \\
1-body & $\mathrm{fp}$ & 6165 & 6186 \\
2-body & $\mathrm{fp}$ & 6187 & 6389 \\
\hline
\end{tabular}

\subsection{Input of type 1}

This type of input allows the user to select a particular use of the RME program. By selecting appropriate data sets of type 1 the user may calculate reduced matrix elements for one or two body operators, in either the $p$, sd, or fp shells, in the FSIF or NPF [8].

The input of type 1 needed for the RME program is identical to the input of type 1 used in the CFP program, and therefore should be obtained from the CPC library as data file $\mathrm{AAC}^{*}$.

Input of type 1 consists of two blocks of input data, the first block to be selected from set $\mathrm{A}$ and the second block from set B.

\subsubsection{Data $\operatorname{set} A$}

Data blocks of set $\mathrm{A}$ contain the necessary information to describe fully the one or two body tensor operators needed.

Since a one (two) body operator is constructed by coupling a one (two) particle creation operator with a one (two) particle destruction operator we can take advantage of the fact that the one (two) particle creation operators are already described by data set A of the CFP program, and we can therefore avoid constructing a new data set $\mathbf{A}$ for the RME program.

It should be pointed out that since a general two-body tensor operator may contain both symmetrically and antisymmetrically coupled pairs, both the symmetric and antisymmetric two-particle operators must be read in and stored.

Table 1 gives the first and last card of data file $\mathrm{AAC}^{*}$ which make up data set $\mathrm{A}$ for a particular application. The meaning of the quantities in set $\mathbf{A}$ is the same as in the CFP program and the reader is referred to ref. [1] for details.

\subsubsection{Data set $B$}

Data blocks of set B contain a table for the reduction $\mathrm{U}(n) \supset \mathrm{SU}(3)$ where $n=3$ for the $\mathrm{p}$ shell, $n=6$ for the sd shell and $n=10$ for the fp shell. These tables are needed in the calculation of highest weight states.

Table 2 gives the first and last card of data file $\mathrm{AAC}^{*}$ which make up data set $\mathrm{B}$ for a given shell. The meaning of the quantities in set B is the same as in the CFP program, and the reader is referred to ref. [1] for details. 
Table 2

\begin{tabular}{llll}
$\begin{array}{l}\text { Space symmetry } \\
\mathrm{U}(n)\end{array}$ & $\begin{array}{l}\text { Restrictions on } \\
\text { SU(3) content }\end{array}$ & First card & Last card \\
\hline $\mathrm{U}(3)$ & none & 6390 & 6399 \\
$\mathrm{U}(6)$ & none & 6400 & 6525 \\
$\mathrm{U}(10)$ & $\alpha_{\max } \leqslant 10$ & 6526 & 7423 \\
\hline
\end{tabular}

\subsection{Input of type 2}

This data must be provided by the user.

Cards $1-5$ define the $\langle\mathrm{BRA}|$ and $|\mathrm{KET}\rangle$ states desired. The meaning of the quantities read in as well as their use in identical with that of ref. [1] except for the following changes:

Card 1: IPRST, IPRLVL, IPRTSK, MPUNCH

FORMAT (1614)

the variable MPUNCH was added to control punching or logical $\mathrm{I} / 0$ unit 7 of the RME.

Card 2: IREPMN, IREPMX, LOHI

the variables IREPMN and IREPMX are not used.

Card 3: ((LINBUF(I,J), I = 1, IMAX), $\mathrm{J}=1,2)$

FORMAT (16I4)

$\mathrm{J}=2$ is not used since we assume that the set of $\langle\mathrm{BRA}|$ states is the same as the set of $|\mathrm{KET}\rangle$ states.

Cards 4-5: same as in the CFP program.

Cards 6-N: JM1(I), MU1(I), IS1(I), IT 1(I), JM2(I), MU2(I), IS2(I), IT2(I), JM3(I), MU3(I), IS3(I), IT3(I)

referring to eq. (5) the meaning of the quantities in cards $6-\mathrm{N}$ is:

$$
\begin{aligned}
& \operatorname{JM} 1(\mathrm{I})=\lambda_{1}, \quad \operatorname{MU1}(\mathrm{I})=\mu_{1}, \quad \operatorname{IS1}(\mathrm{I})=S_{1}, \quad \operatorname{IT} 1(\mathrm{I})=T_{1}, \quad \mathrm{JM} 2(\mathrm{I})=\mu_{2}, \quad \operatorname{MU} 2(\mathrm{I})=\lambda_{2}, \quad \operatorname{IS} 2(\mathrm{I})=S_{2}, \\
& \operatorname{IT} 2(\mathrm{I})=T_{2}, \quad \mathrm{JM} 3(\mathrm{I})=\lambda, \quad \mathrm{MU} 3(\mathrm{I})=\mu, \quad \operatorname{IS} 3(\mathrm{I})=S, \quad \operatorname{IT} 3(\mathrm{I})=T,
\end{aligned}
$$

If the NPF is being used one should set IT1(I) $=$ IT2(I) $=$ IT3(I) $=0$.

\subsection{Output}

The output is controlled by the value of the variables read-in in card 1 as follows:

IPRST $=0$ : no printing of the many-particle states,

$=1$ : print the many-particle states.

IPRLVL $=0$ : no printing of single particle levels ,

$=1$ : print single particle levels,

$=2$ : print both the single particle levels and the explicit forms of raising and lowering operators.

IPRTSK $=0$ : no printing of summary of states,

$=1$ : print a summary of states to be calculated.

MPUNCH $=0$ : no punching of the RME,

$=1$ : punch-out the RME as well as the many-particle states on logical $\mathrm{I} / 0$ unit 7.

\section{Program modifications}

The size of arrays needed to calculate the $\langle\mathrm{BRA}|$ and $|\mathrm{KET}\rangle$ states depend strongly on the nuclear shell under consideration as well as the number of particles involved. Therefore provision is made for easy adjustment of 
Table 3

\begin{tabular}{llrrr}
\hline CSECT & Parameter & \multicolumn{3}{l}{ Oscillator shell } \\
\cline { 3 - 5 } & & $\mathrm{p}$ & \multicolumn{1}{c}{$\mathrm{sd}$} & \multicolumn{1}{c}{$\mathrm{fp}$} \\
\hline CTOP & NP21 & 50 & 100 & 200 \\
RME & NS7 & 66 & 276 & 780 \\
& NS8 & 12 & 24 & 40 \\
& NP22 & 500 & 1500 & 3750 \\
& NP23 & 500 & 1000 & 2000 \\
\hline
\end{tabular}

dimensions in accordance with the needs of a particular user.

Instructions for the proper dimensioning of arrays needed in subroutines of blocks 2 and 3 can be found in ref. [1] and ref. [2], respectively.

The procedure for dimensioning the arrays which appear in the subroutines of block 1 is similar to the one adopted in the CFP program. Table 3 gives the suggested value for the parameters which determine the size of arrays in those labelled COMMON blocks which do not appear in the CFP program. In the event that the dimension is insufficient a relevant message will be printed asking the user to increase its value and execution is stopped.

As with the CFP program, considerable saving of high speed storage (up to $35 \%$ ) can be achieved by using half-length words for all integer arrays which begin with the letter "L". In IBM 360/370 or similar operating systems this can be done by simply inserting as the first statement of each subroutine IMPLICIT INTEGER*2(L).

\section{Test run}

The RME program has been extensively tested by checking against RME sum rules. We reproduce below a test run, as well as, the necessary input data required.

In this test run we calculate RME in the FSIF for two-body operators in the fp shell.

The set of $\langle\mathrm{BRA}|$ and $|\mathrm{KET}\rangle$ states are all the states of the form:

$$
\left|n=6(\lambda \mu) \alpha\left(\mathrm{PP}^{\prime} \mathrm{P}^{\prime \prime}\right) \beta S=\mathrm{P} T=\mathrm{P}^{\prime}\right\rangle
$$

with $2 \lambda+\mu=30$ and $2 \lambda+\mu=27$ the two-body tensor operators are:

$$
\begin{aligned}
& {\left[\left[a^{+} a^{+}\right]^{(60) 20}[a a]^{(06) 20}\right]^{(00) 00},\left[\left[a^{+} a^{+}\right]^{(22) 20}[a a]^{(22) 02}\right]^{(22) 22},} \\
& {\left[\left[a^{+} a^{+}\right]^{(41) 22}[a a]^{(22) 20}\right]^{(25) 42},\left[\left[a^{+} a^{+}\right]^{(03) 22}[a a]^{(14) 22}\right]^{(17) 44} .}
\end{aligned}
$$

The input deck required for this run is:
DATA SET A: 2-body fp shell
CARDS $1909-2881$
DATA SET B: $\quad \mathrm{U}(10) \supset \mathrm{SU}(3)$
CARDS $6526-7423$
CARD 1: 1111110
FORMAT (16I4)
CARD 2: 0000
FORMAT (16I4)
CARD 3: $6-222-111-111-111-111-333-333$
FORMAT (16I4)
CARD 4: 23027
FORMAT (20I4)
CARD 5: $\quad \begin{array}{llllllllllll}6 & 6 & 2 & 0 & 0 & 6 & 2 & 0 & 0 & 0 & 0 & 0\end{array}$
FORMAT (16I4)
CARD 6: $\begin{array}{lllllllllllll}2 & 2 & 2 & 0 & 2 & 2 & 0 & 2 & 2 & 2 & 2 & 2\end{array}$
FORMAT (16I4)
CARD 7: $\quad \begin{array}{llllllllllll}4 & 1 & 2 & 2 & 2 & 2 & 2 & 0 & 2 & 5 & 4 & 2\end{array}$
CARD 8: $\begin{array}{lllllllllllll}0 & 3 & 2 & 2 & 1 & 4 & 2 & 2 & 1 & 7 & 4 & 4\end{array}$
FORMAT (16I4)
FORMAT (16I4) 


\section{Acknowledgements}

It is a pleasure to acknowledge numerous enlightening discussions with Prof. K.T. Hecht.

\section{References}

[1] D. Braunschweig, Comput. Phys. Commun. 14 (1978) 109.

[2] Y. Akiyama and J.P. Draayer, Comput. Phys. Commun. 5 (1973) 405.

[3] J.B. French, E.C. Halbert, J.B. McGrory and S.S.M. Wong, Adv. in Nucl. Phys., vol. 3; R.R. Whitehead, A. Watt, B.J. Cole and I. Morrison, Adv. in Nucl. Phys., vol. 9; R. Gross and Y. Accad, Comput. Phys. Commun. 8 (1974) 101.

[4] S. Cohen and D. Kurath, Nucl. Phys. 73 (1963) 1;

[5] B.J. Cole, D. Kelvin, A. Watt and R.R. Whitehead, J. Phys. G3 (1977) 919;

J.B. McGrory, Phys. Rev. 160 (1967) 915; J.E. Koops and P.W.W. Glausemans, Z. Physik (in print); A. Arima and D. Strottman, Oxford Univ., Nucl. Phys., Report No. 46; J.F. Van Hienen, W. Chung and B.H. Wildenthal, Nucl. Phys. A269 (1976) 159.

[5] M. Harvey, Adv. in Nucl. Phys. 1 (1968) 67; D.J. Millener, Proc. Seminar in nuclear theory, Queen's Univ. Kingston, Canada (1976); M. Conze, H. Feldmeier and P. Manakos, Phys. Lett. 43B (1973) 101;

Y. Akiyama, A. Arima and T. Sebe, Nucl. Phys. A138 (1969) 273;

P.J. Ellis and J. Engeland, Nucl. Phys. A144 (1970) 161.

[6] T. Inoue, Y. Akiyama and K. Shirai, Proc. of the Inst. of Nat. Sci. Nihon Univ. 12 (1977); K.T. Hecht, Phys. Lett. 58B (1975) 252; R.D. Ratna Raju, M. Satyanarayana Reddi and V.K.B. Kota, J. Phys. G3 (1977) 1063.

[7] R.D. Ratna Raju, J.P. Draayer and K.T. Hecht, Nucl. Phys. A202 (1973) 433.

[8] The code which calculates the input data of type 1 for higher shells will be made available soon. 


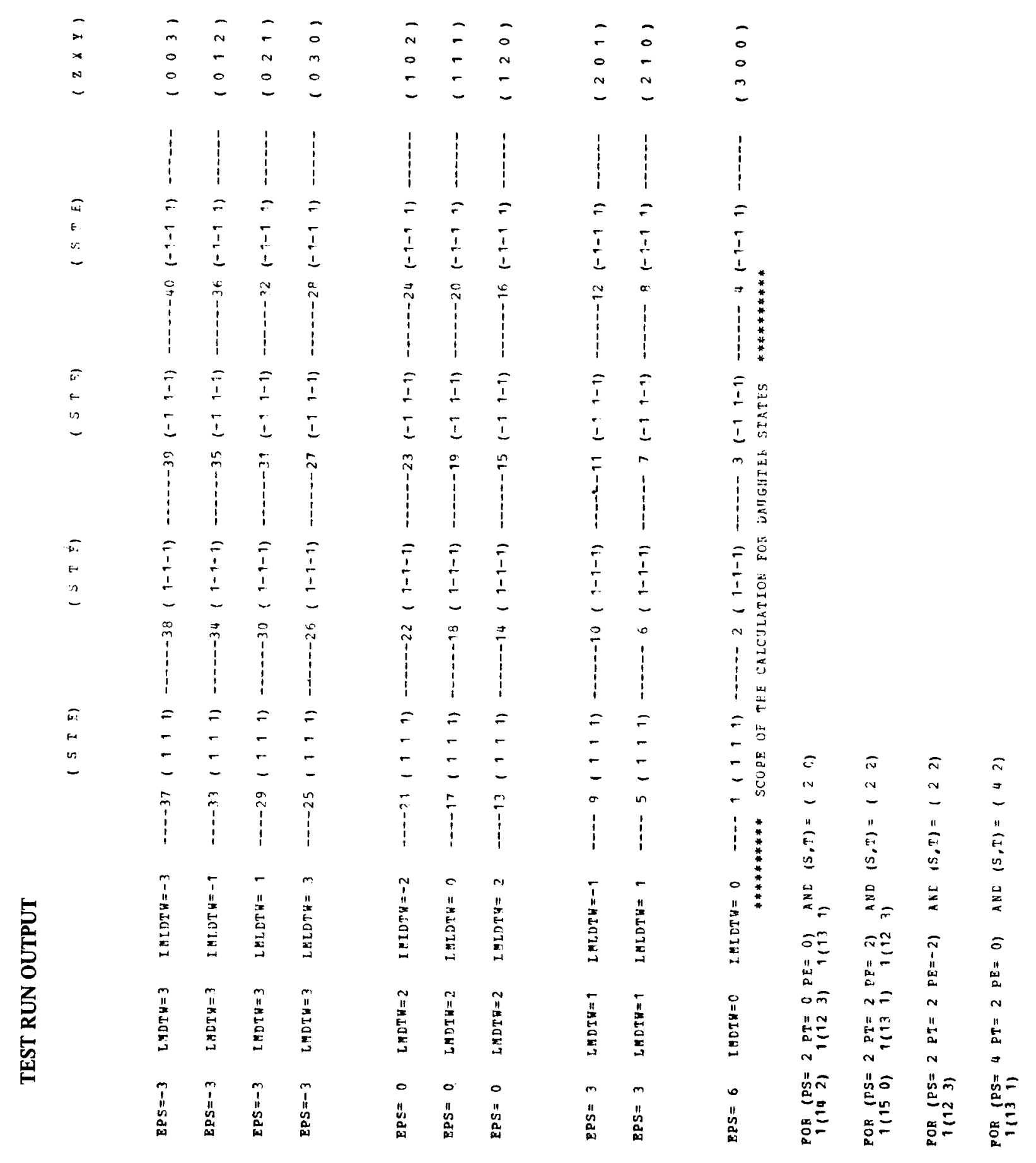




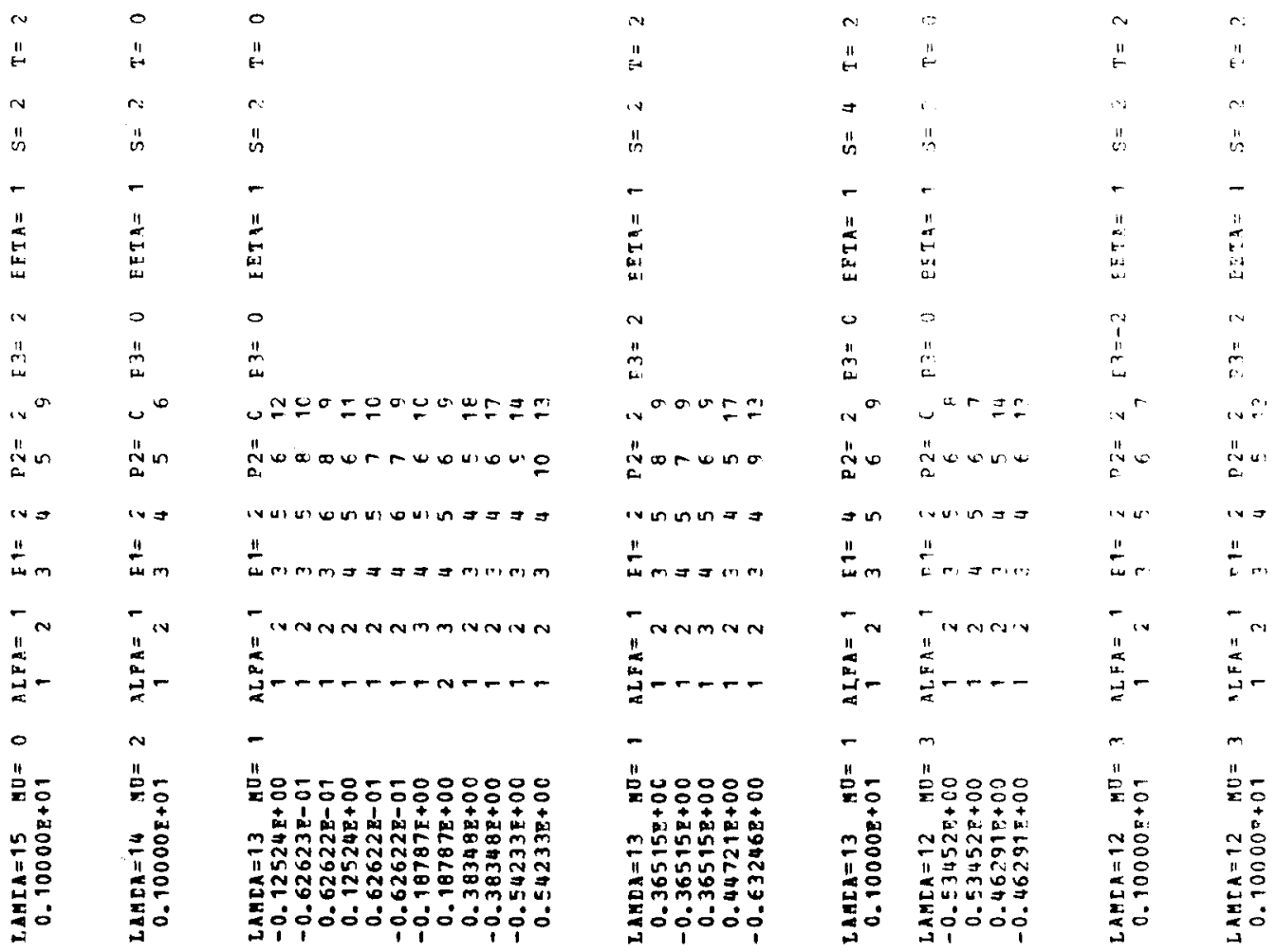




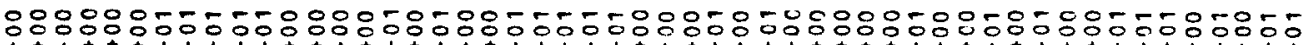

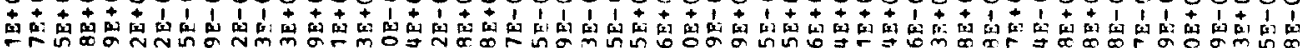

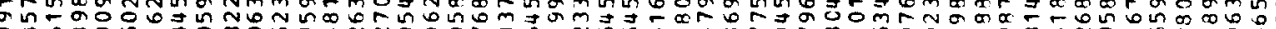

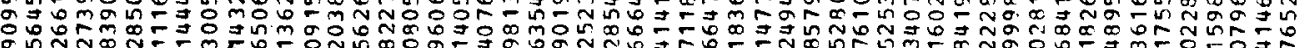
品

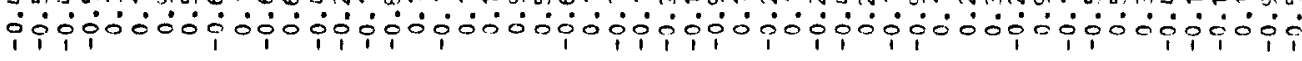

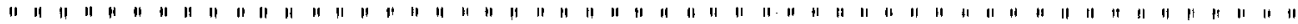

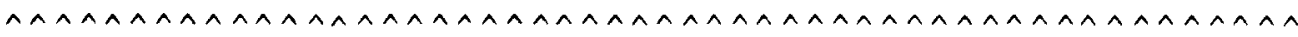

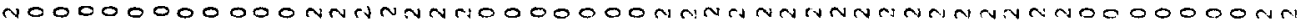

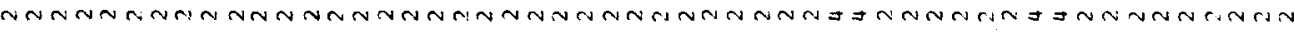

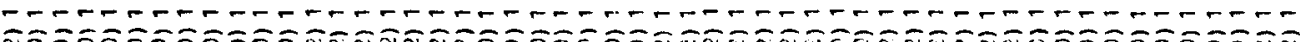
तо

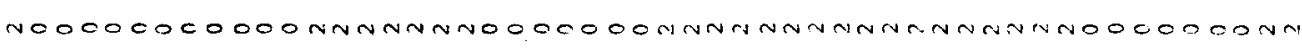

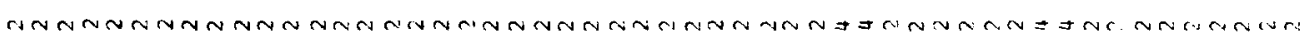

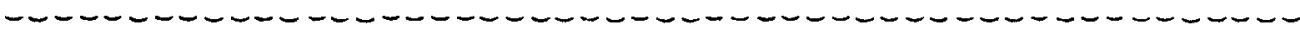

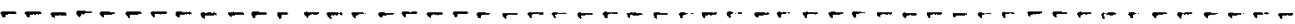

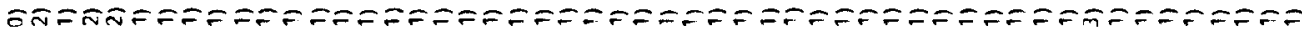

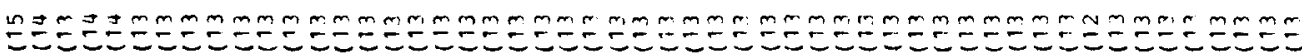

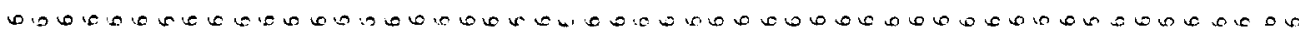

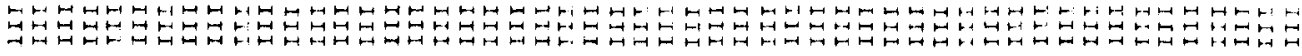

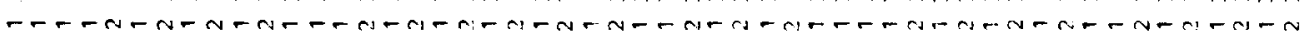

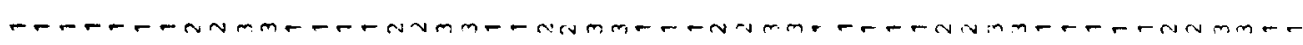

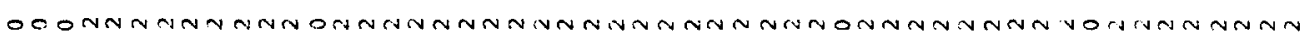

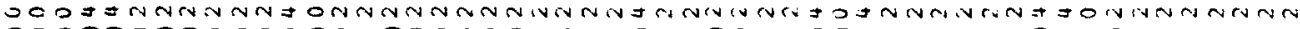

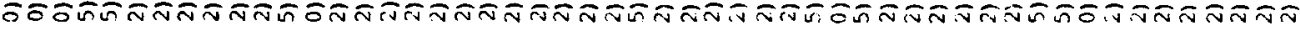

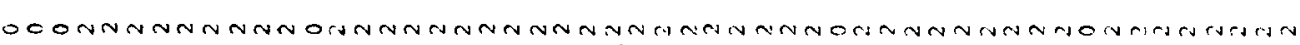

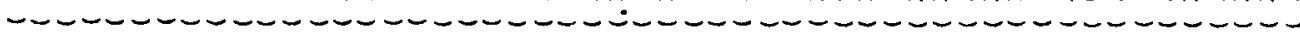

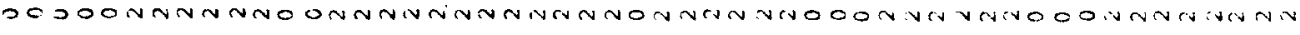

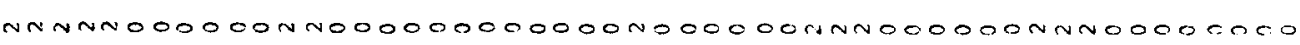
-

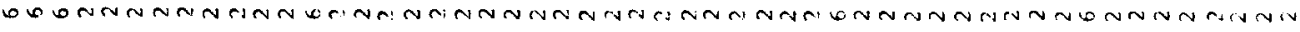

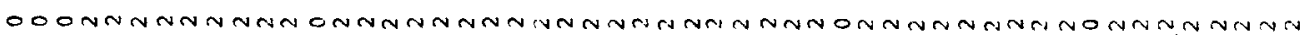

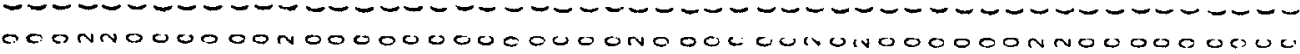

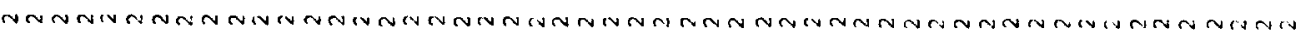
-

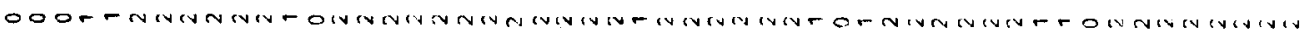

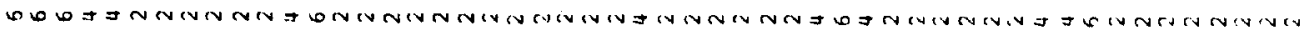

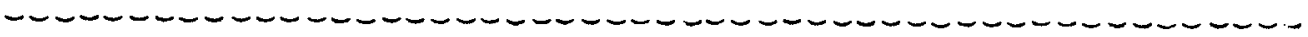

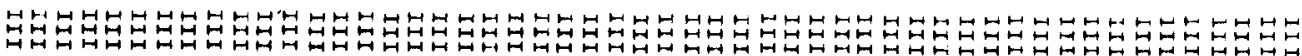
no onn

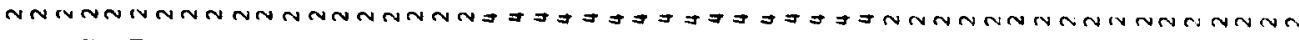

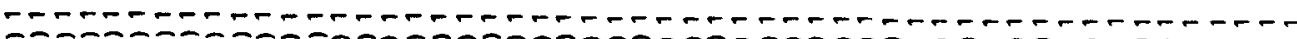

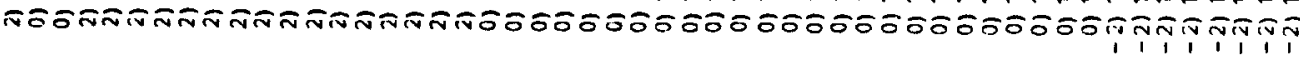

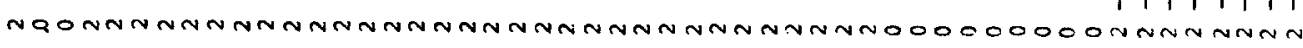

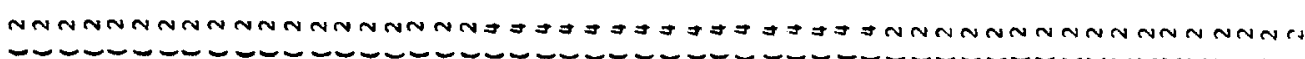

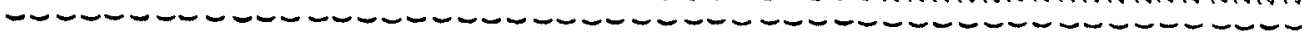

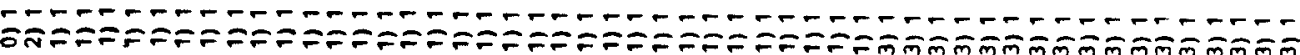

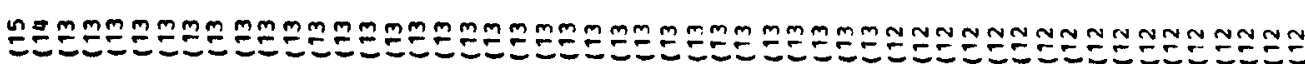

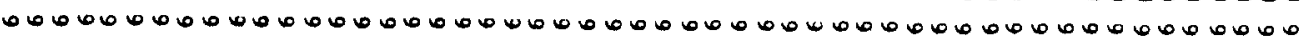

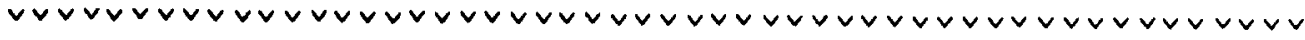




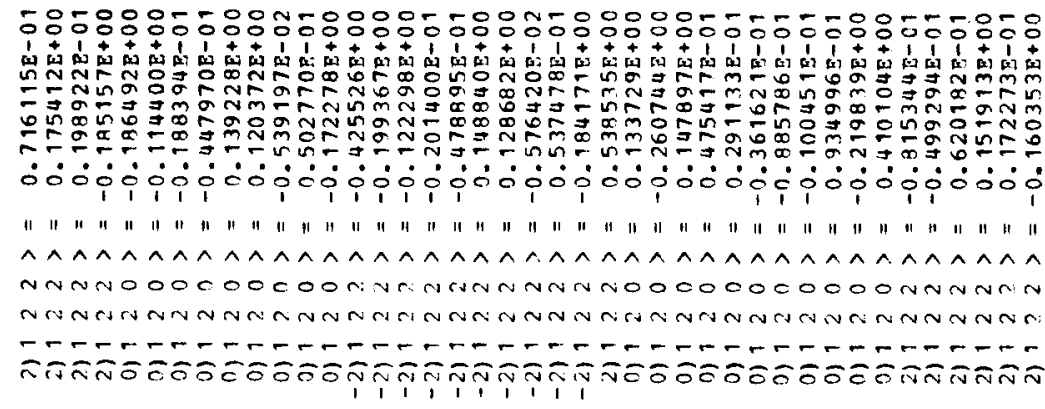

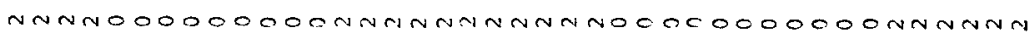

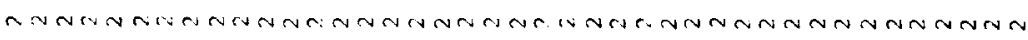
- - - - - - - - - - - - - - -

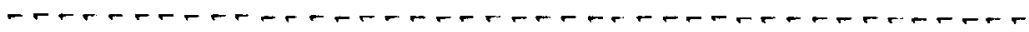

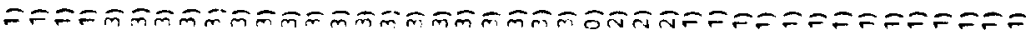

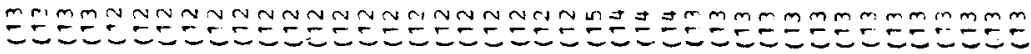

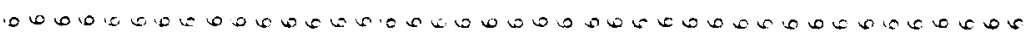

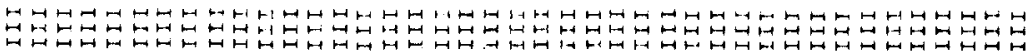

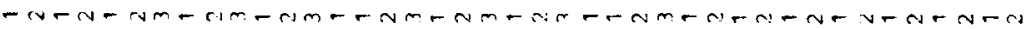

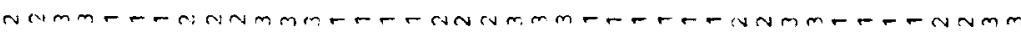

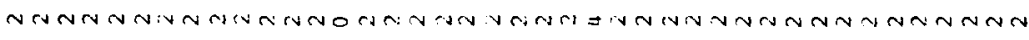

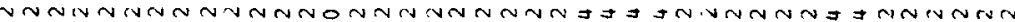

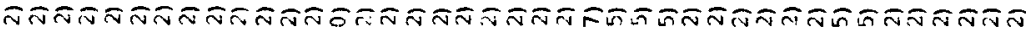

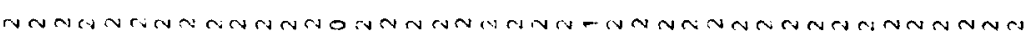

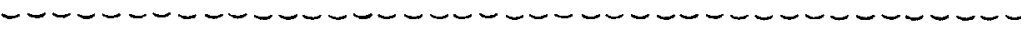

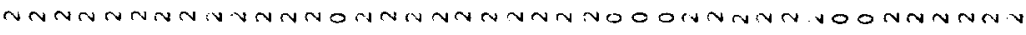

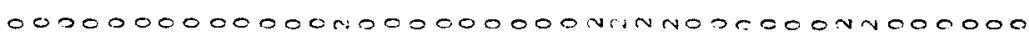

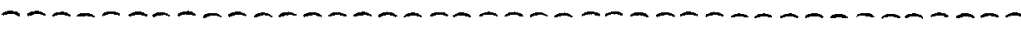

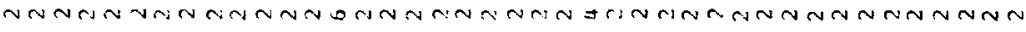

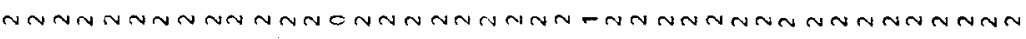
$00000000000000000000000 n n N m 000000 n n c 00000$

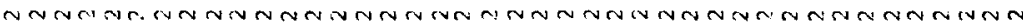
-

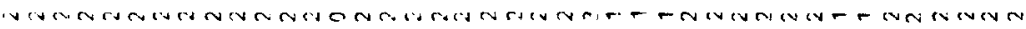

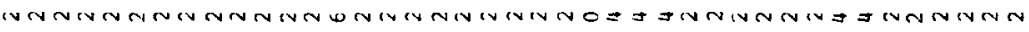

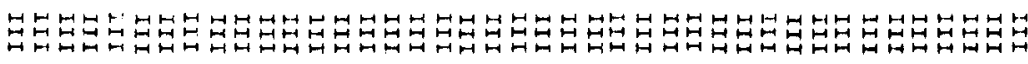

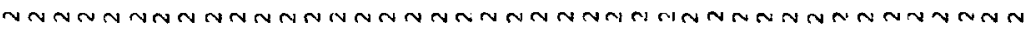

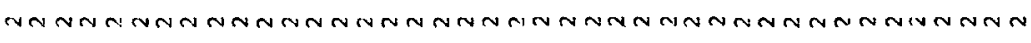

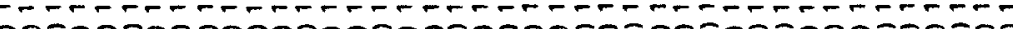

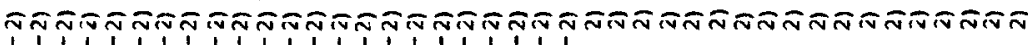

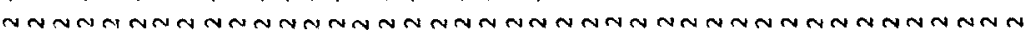

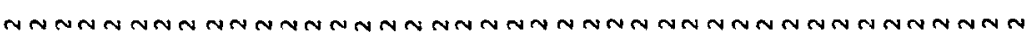

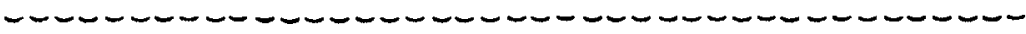

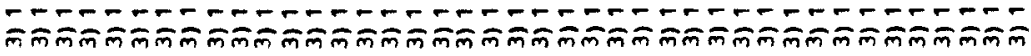

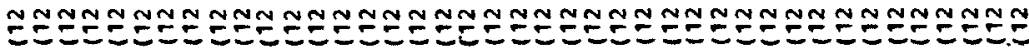

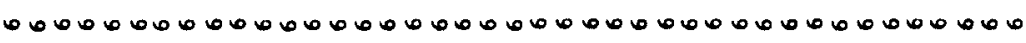

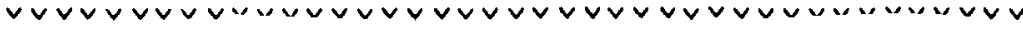




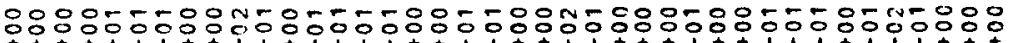

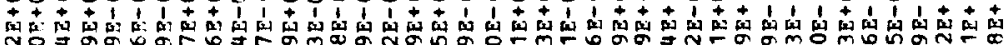

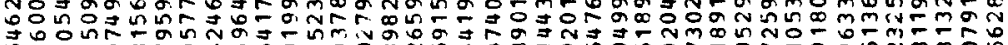

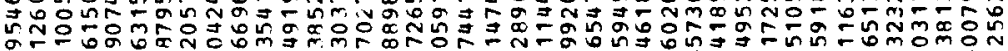

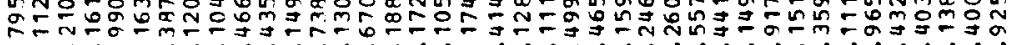

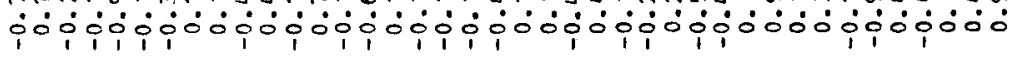

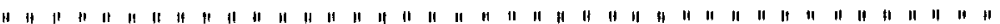
$\wedge \wedge \wedge \wedge \wedge \wedge \wedge \wedge \wedge \wedge \wedge \wedge \hat{\wedge} \wedge \wedge \wedge \wedge \wedge \wedge \wedge \wedge \wedge \wedge \wedge \wedge \wedge \wedge \wedge \wedge \wedge \wedge \wedge \wedge \wedge \wedge \wedge \wedge \wedge \wedge$

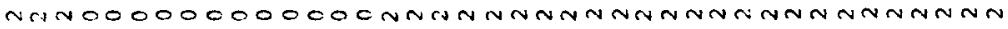

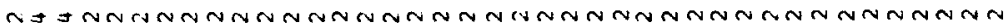

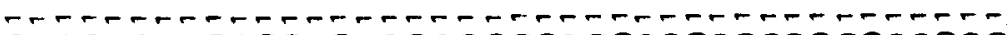

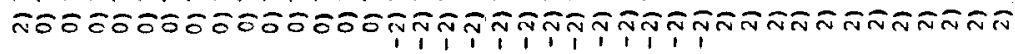

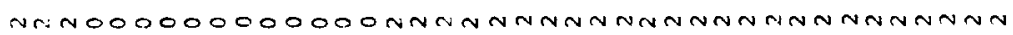

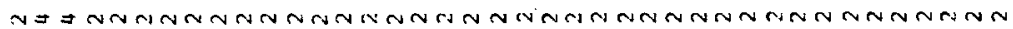

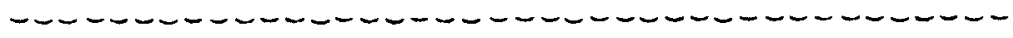

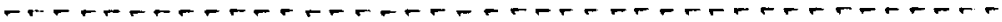

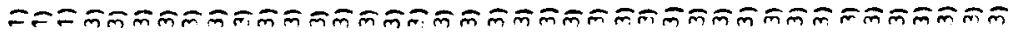

MEI

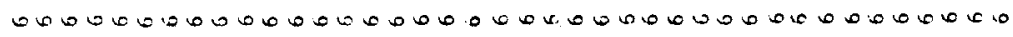

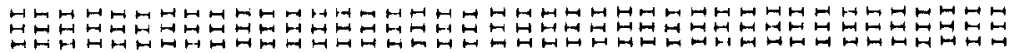

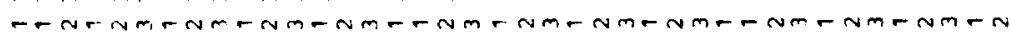

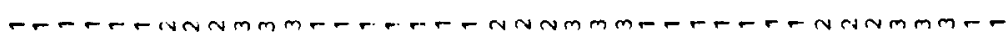

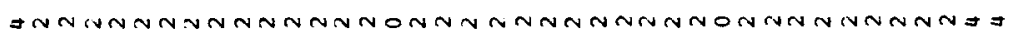

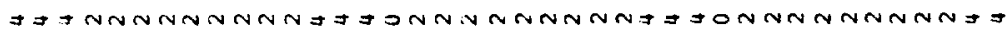

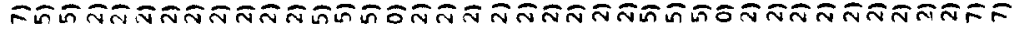

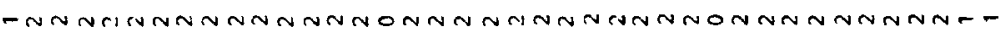

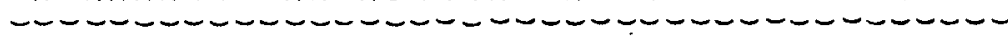
no oinnwranninn

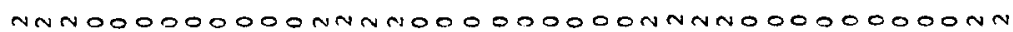
- - - - - - - - - - - - - - - - - - - - - - - - - - - - - JnN

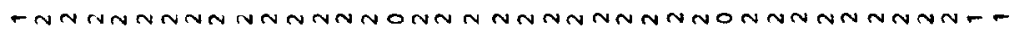

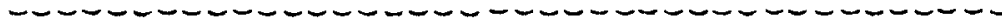

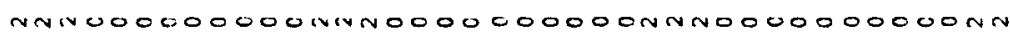

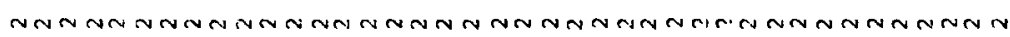

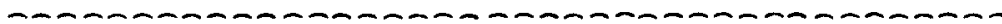

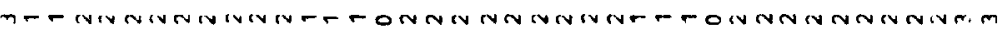

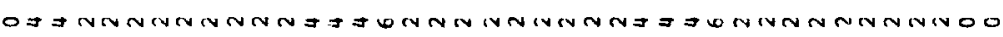

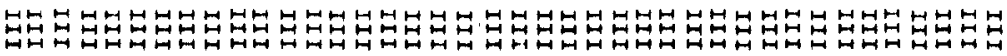

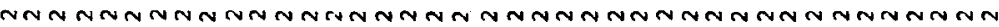

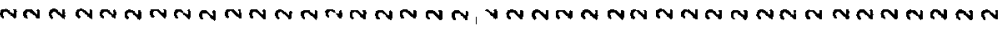
-

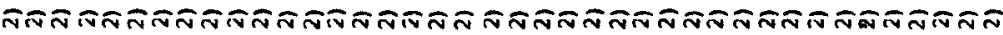

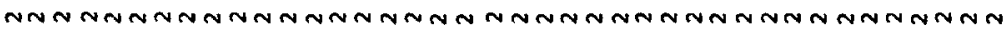

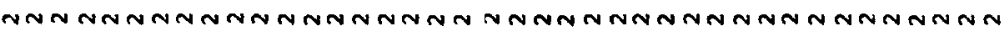

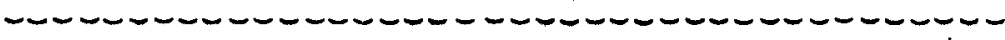

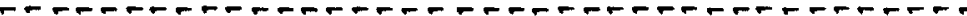

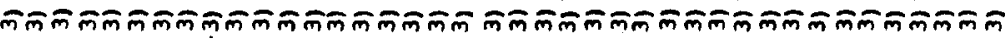

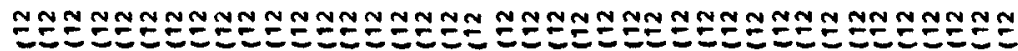
๑

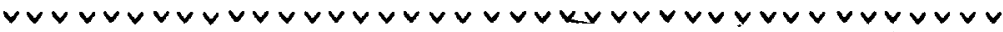

